\title{
Hydrogen Sulfide Exerts Anti-oxidative and Anti-inflammatory Effects in Acute Lung Injury
}

\author{
Kornelia K. Zimmermann, ${ }^{1}$ Sashko G. Spassov, ${ }^{1}$ Karl M. Strosing, ${ }^{1}$ Paul M. Ihle, ${ }^{1}$ \\ Helen Engelstaedter, ${ }^{1}$ Alexander Hoetzel, ${ }^{1}$ and Simone Faller $\mathbb{i}^{1,2}$
}

\begin{abstract}
Acute lung injury (ALI) caused by septic stimuli is still a major problem in critical care patients. We have shown previously that hydrogen sulfide $\left(\mathrm{H}_{2} \mathrm{~S}\right)$ mediates anti-inflammatory and lung protective effects. In the present study, we aimed to investigate the underlying mechanisms. C57BL/6N mice were instilled with lipopolysaccharide (LPS) intranasally in the absence or presence of inhaled $\mathrm{H}_{2} \mathrm{~S}$ for $6 \mathrm{~h}$. LPS instillation led to alveolar wall thickening, an elevated ALI score, increased neutrophil transmigration, and elevated interleukin-1 $\beta$ cytokine release into the bronchoalveolar lavage fluid. In contrast, $\mathrm{H}_{2} \mathrm{~S}$ inhalation prevented lung injury and inflammation despite LPS treatment. Moreover, $\mathrm{H}_{2} \mathrm{~S}$ inhalation significantly inhibited protein expression of cystathionine- $\beta$-synthetase, heat shock protein 70 , phosphorylated p38 MAP kinase, NADPH oxidase 2, and the formation of reactive oxygen species (ROS) in LPS-challenged animals. In conclusion, $\mathrm{H}_{2} \mathrm{~S}$ prevents LPS-induced ALI by inhibition of pro-inflammatory and oxidative responses via the concerted attenuation of stress protein, MAP kinase, and ROS signaling pathways.
\end{abstract}

KEY WORDS: acute lung injury; hydrogen sulfide; lipopolysaccharide; NADPH oxidase 2; reactive oxygen species.

\section{BACKGROUND}

Pulmonary infection is the most important cause of acute lung injury (ALI) and acute respiratory distress syndrome (ARDS) and continues to produce a high rate of morbidity and mortality in the intensive care unit [1]. Lung injury upon infection is characterized by a strong inflammatory response, reflected by transmigration of immune-competent cells (e.g., neutrophils) and the release of pro-inflammatory cytokines

\footnotetext{
${ }^{1}$ Department of Anesthesiology and Critical Care Medicine, Medical Center - University of Freiburg, Faculty of Medicine, University of Freiburg, Hugstetter Str. 55, 79106 Freiburg, Germany

${ }^{2}$ To whom correspondence should be addressed at Department of Anesthesiology and Critical Care Medicine, Medical Center - University of Freiburg, Faculty of Medicine, University of Freiburg, Hugstetter Str. 55, 79106 Freiburg, Germany. E-mail: simone.faller@uniklinik-freiburg.de
}

(e.g., interleukin-1 $\beta$ ). Subsequently, the inflammatory process damages alveolar architecture resulting in critical impairment of lung function [2]. Despite antibiotic treatment and protective mechanical ventilation, to date no specific treatment has been shown to improve the clinical outcome of patients suffering from ALI/ARDS.

Several signaling pathways are involved in the outcome of ALI caused by infective stimuli. Among them, the induction of heat shock proteins like hemeoxygenase-1 (HO-1) and heat shock protein 70 (HSP70) [3], or the inhibition of MAP kinase signaling, all mediate cytoprotective and anti-inflammatory effects in various models of lung injury [4-6]. In addition, the inflammatory response in sepsis is also triggered by oxidative stress resulting from the excessive production of reactive oxygen species (ROS). In this respect, hydrogen sulfide $\left(\mathrm{H}_{2} \mathrm{~S}\right)$ is an 
endogenously produced gasotransmitter that is known for its anti-inflammatory and redox regulating capacity. Exogenous administration of $\mathrm{H}_{2} \mathrm{~S}$ has been shown to mediate protective effects in various animal disease models, such as ischemia-reperfusion injury [7], ventilator-induced ALI [8], hyperoxia-induced ALI [9], or oleic acid-induced lung injury [10]. We have recently demonstrated that inhalation of 80 parts per million (ppm) $\mathrm{H}_{2} \mathrm{~S}$ exerts anti-inflammatory effects and can protect against ALI in an inflammatory model of lipopolysaccharide (LPS)-induced lung injury [11]. However, the molecular signaling pathways involved in the observed protection are widely unknown. We therefore hypothesized that $\mathrm{H}_{2} \mathrm{~S}$ may modulate the generation of ROS and that $\mathrm{H}_{2} \mathrm{~S}$ producing enzymes, heat shock protein responses, MAP kinase signaling, and the release and attraction of inflammatory mediators may also be affected by $\mathrm{H}_{2} \mathrm{~S}$ in LPS-induced ALI. In the present study, we thus defined the role of antiinflammatory, anti-oxidative, and feedback pathways in $\mathrm{H}_{2} \mathrm{~S}$-mediated lung protection during inflammation. We show for the first time that prevention of lung injury is associated with the anti-oxidative effects of $\mathrm{H}_{2} \mathrm{~S}$ inhalation leading to a concerted inhibition of $\mathrm{p} 38$ MAPK signaling, ROS formation, and Nox 2 expression.

\section{METHODS}

\section{Animals and Experimental Setting}

C57BL/6N mice $(n=24)$ were randomly assigned to four experimental groups: Group 1 (control): mice were exposed to synthetic air for $1 \mathrm{~h}$, were instilled with $70 \mu \mathrm{l}$ endotoxin-free PBS intranasally (i.n.), and breathed air for another $6 \mathrm{~h}$. Group 2 (control $+\mathrm{H}_{2} \mathrm{~S}$ ): mice breathed air supplemented with $80 \mathrm{ppm}_{2} \mathrm{~S}$ for $1 \mathrm{~h}$, received PBS i.n., and breathed $\mathrm{H}_{2} \mathrm{~S}$ for another $6 \mathrm{~h}$. Group 3 (LPS): mice breathed air for $1 \mathrm{~h}$, received $0.25 \mathrm{ng}$ LPS in $70 \mu \mathrm{l}$ PBS i.n. (Escherichia coli 055:B5; Sigma-Aldrich Chemie GmbH, Munich, Germany), and breathed air for another $6 \mathrm{~h}$. Group 4 (LPS $+\mathrm{H}_{2} \mathrm{~S}$ ): mice breathed $80 \mathrm{ppm} \mathrm{H}_{2} \mathrm{~S}$ for $1 \mathrm{~h}$, received LPS i.n., and breathed $\mathrm{H}_{2} \mathrm{~S}$ for another $6 \mathrm{~h}$. Experiments were performed in a sealed Plexiglass chamber in which $\mathrm{H}_{2} \mathrm{~S}$ concentration was measured continuously using a portable gas monitor (MX6 iBrid, Industrial Scientific Corporation, Oakdale, PA). All animal experiments were performed in accordance with guidelines of the local animal care commission (Ethics Committee University of Freiburg and Regierungspräsidium Freiburg, Freiburg, Germany, Permission No. G-07/25) and in conformance with the journals' requirements for human and animal trials.

\section{End of Experiment, Tissue Sampling, and Bronchoalveolar Lavage}

At the end of each experiment, all mice were sacrificed by an intraperitoneal, overdosed injection of ketamine $(180 \mathrm{mg} / \mathrm{kg})$ and acepromazine $(1.8 \mathrm{mg} / \mathrm{kg})$. Bronchoalveolar lavage (BAL) fluid and lung tissue for Western Blot analysis and histological examination were gained as described previously $[9,11]$.

\section{Cytokine Measurements}

BAL aliquots were analyzed using an interleukin- $1 \beta$ (IL-1 $\beta$ ) ELISA kit (R\&D Systems GmbH, Wiesbaden, Germany) according to the manufacturers' instructions.

\section{Histological Examination}

Cryosections of the left lung lobes and hematoxylin and eosin staining was performed and analyzed as described previously [9].

\section{Detection of Reactive Oxygen Species}

Dihydroethidium (DHE, Life Technologies GmbH, Darmstadt, Germany) was used to detect reactive oxygen species (ROS) in lung tissue as described earlier [9]. Densitometric analysis was performed with the ImageJ software (National Institutes of Health; Bethesda, MD; available at: http://rsbweb.nih.gov.).

\section{Immunoblotting}

Western blotting was performed as described recently [12]. The membranes were incubated with antibodies against 3-mercaptopyruvate sulfurtransferase (3MST; Sigma, Taufkirchen, Germany), cystathionine- $\gamma-$ lyase (CSE; Santa Cruz Biotechnology Inc., Heidelberg, Germany) or cystathionine- $\beta$-synthetase (CBS; Abnova, Heidelberg, Germany), hemeoxygenase-1 (HO-1; Biomol GmbH, Hamburg, Germany), heat shock protein 70 (HSP70; Abcam, Cambridge, UK), phosphorylated c-jun N-terminale kinase (pJNK), phosphorylated extracellular-signal regulated kinases (pERK1/2), phosphorylated p38 (pp38; all Cell Signaling, Frankfurt, Germany), NADPH oxidase 1 and 4 
(Nox1; Nox4; Santa Cruz), or Nox2 (Becton Dickinson $\mathrm{GmbH}$, Heidelberg, Germany). Normalization in order to control equal protein loading was performed by stripping and re-blotting of the membranes with glyceraldehyde-3-phosphate-dehydrogenase (GAPDH; Enzo Life Sciences GmbH, Lörrach, Germany) or $\beta$ tubulin (Cell Signaling). Data represent fold induction of indicated proteins with respect to GAPDH or $\beta$ tubulin after densitometric analysis.

\section{Statistical Analysis}

Experiments were performed with $n=6$ mice per group. Power calculations were performed prior to the study in order to define group sizes. Graphs represent means \pm standard error of means (SEM). Data were further analyzed for normal variation prior to one-way analysis of variance (ANOVA) followed by the Student-Newman-Keuls post hoc test. In the case of failed normality testing, ANOVA on ranks (Kruskal-Wallis test) was used, followed by the Dunn's post hoc test. $P<0.05$ was considered significant. All calculations were performed with the
SigmaPlot 11.0 statistical software (Systat Software Inc., Erkrath, Germany).

\section{RESULTS}

\section{Effect of Hydrogen Sulfide on LPS-Induced Lung Damage and Inflammation}

When compared to control animals in the presence or absence of $\mathrm{H}_{2} \mathrm{~S}$ (Fig. 1a "control," "control $+\mathrm{H}_{2} \mathrm{~S}$ "), intranasal application of LPS led to a profound induction of histological lung damage after 6 h (Fig. 1a "LPS"), characterized by alveolar wall thickening and an increased ALI score (Fig. 1b, c). In contrast, inhalation of $80 \mathrm{ppm}$ $\mathrm{H}_{2} \mathrm{~S}$ for $6 \mathrm{~h}$ reduced these signs of histopathological damage despite LPS application (Fig. 1a-c). LPS treatment also induced neutrophil sequestration (Fig. 2a) and the release of the pro-inflammatory cytokine IL-1 $\beta$ (Fig. 2b) into the bronchoalveolar space compared to control or control $+\mathrm{H}_{2} \mathrm{~S}$ mice. Likewise, $\mathrm{H}_{2} \mathrm{~S}$ inhalation during LPS treatment reduced neutrophil influx and IL-1 $\beta$ release to control levels (Fig. 2).
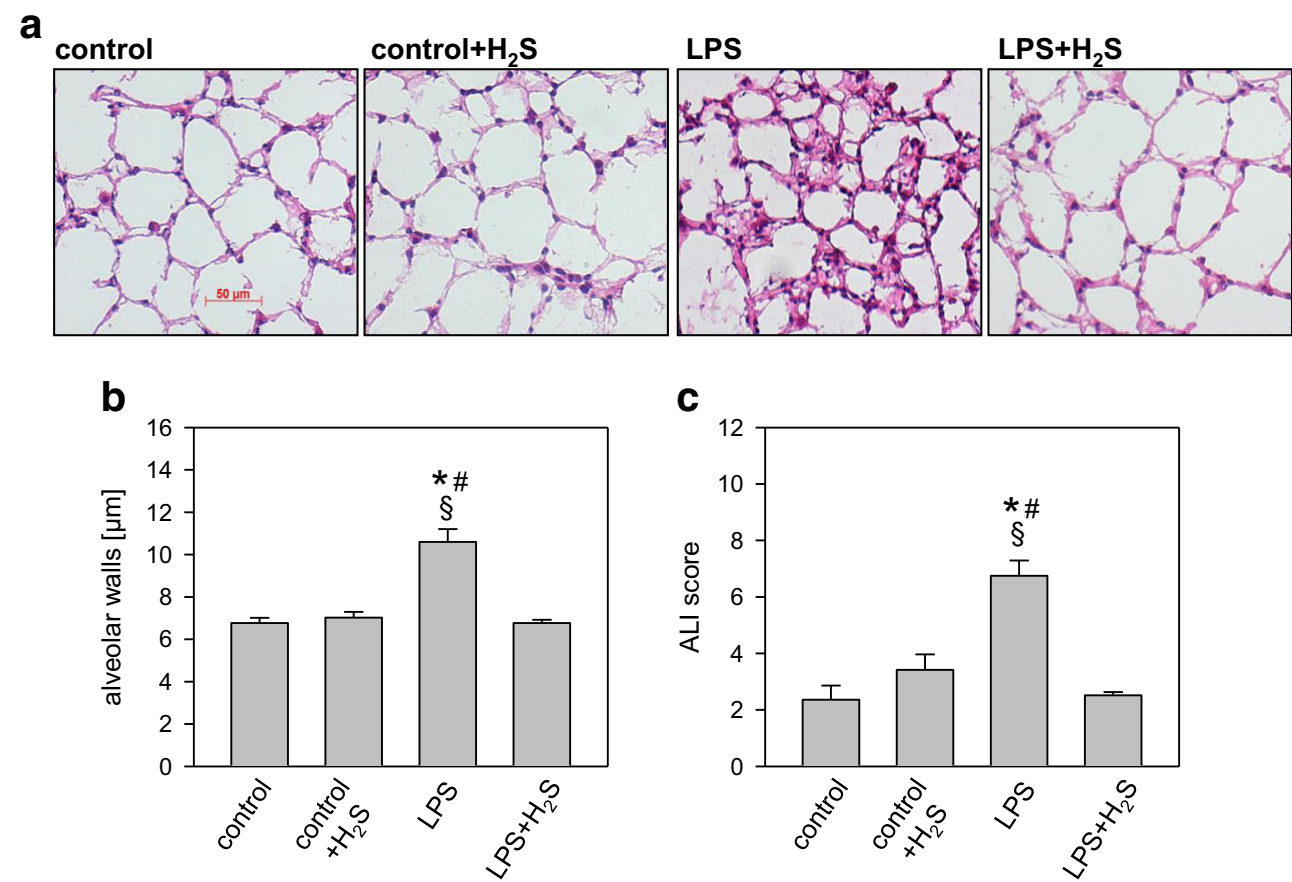

Fig. 1. Effect of hydrogen sulfide on LPS-induced lung damage. As controls, mice received phosphate-buffered saline (PBS, intranasally) and were kept in room air (control) or in $80 \mathrm{ppm} \mathrm{H}_{2} \mathrm{~S}$ (control $+\mathrm{H}_{2} \mathrm{~S}$ ) for $6 \mathrm{~h}$. LPS-treated mice (LPS i.n.) were either kept in room air (LPS) or in $80 \mathrm{ppm} \mathrm{H}_{2} \mathrm{~S}\left(\mathrm{LPS}+\mathrm{H}_{2} \mathrm{~S}\right.$ ) for $6 \mathrm{~h}$. Sections from the left lung lobe were stained with hematoxylin and eosin. Representative pictures are shown for each experimental group (magnification $=200 \times(\mathbf{a})$ ). High-power fields were randomly assigned to measure alveolar wall thickness $(\mathbf{b})$ and to calculate an acute lung injury (ALI) score (c). Data represent means \pm SEM for $n=6$ /group. ANOVA (Student-Newman-Keuls post hoc test), ${ }^{*} P<0.05$ vs. control group; ${ }^{\#} P<0.05 v s$. control + $\mathrm{H}_{2} \mathrm{~S}$ group; ${ }^{\S} \mathrm{P}<0.05$ vs. LPS $+\mathrm{H}_{2} \mathrm{~S}$ group. 
a

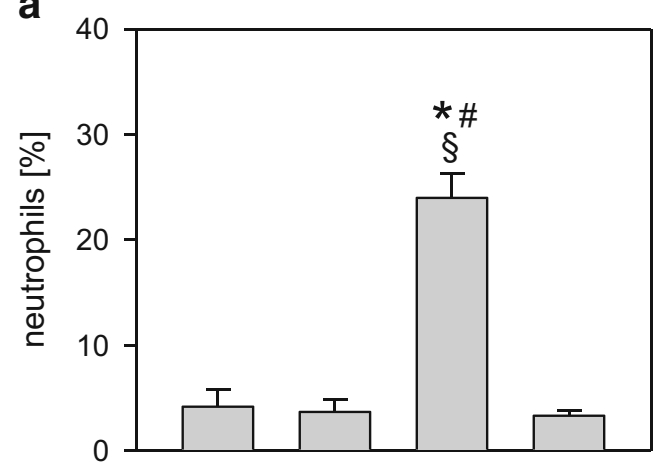

b

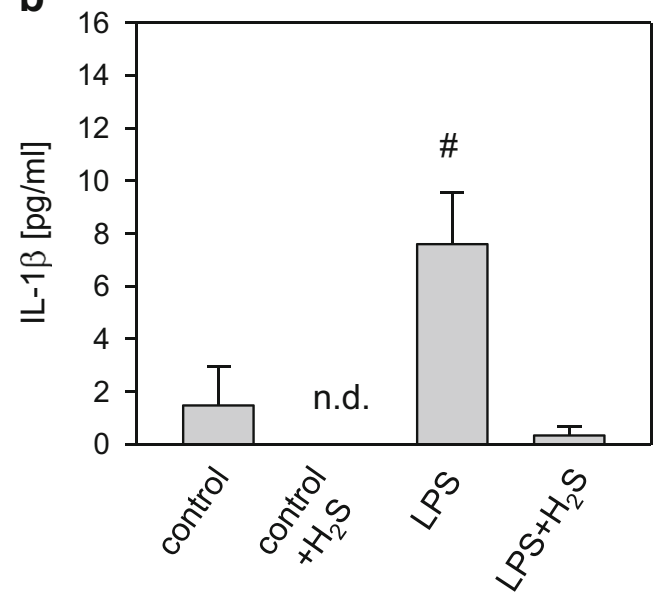

a

3-MST

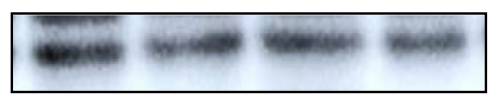

$\beta$-tubulin

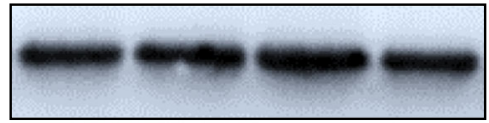

b

CSE

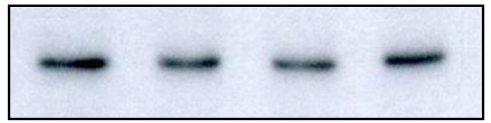

GAPDH

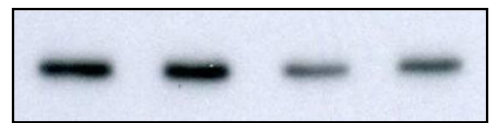

C CBS

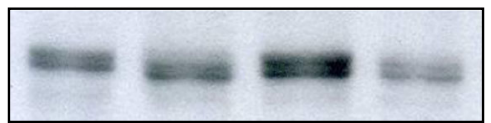

GAPDH

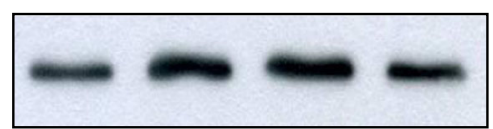

d

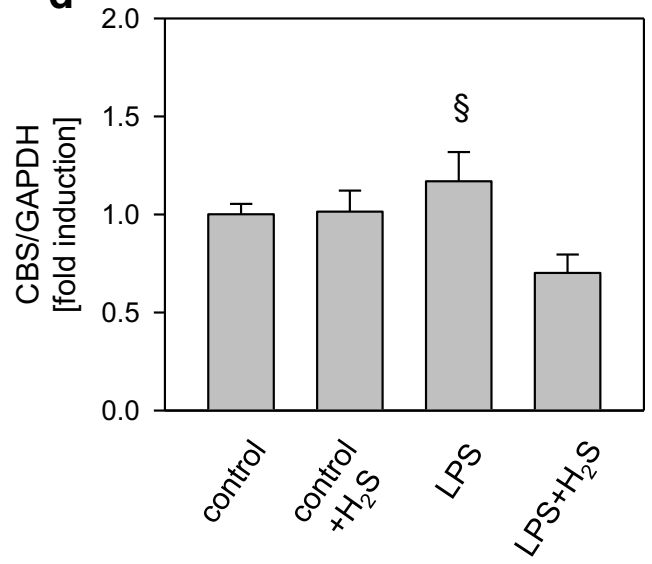

Fig. 3. Effect of hydrogen sulfide on CBS protein expression. As controls, mice received phosphate-buffered saline (PBS, intranasally) and were kept in room air (control) or in $80 \mathrm{ppm} \mathrm{H}_{2} \mathrm{~S}$ (control $+\mathrm{H}_{2} \mathrm{~S}$ ) for $6 \mathrm{~h}$. LPS-treated mice (LPS i.n.) were either kept in room air (LPS) or in $80 \mathrm{ppm}_{2} \mathrm{~S}$ (LPS $+\mathrm{H}_{2} \mathrm{~S}$ ) for $6 \mathrm{~h}$. Lung samples were taken from the upper right lobe for Western blot analysis. Normalization in order to control equal protein loading was performed by stripping and re-blotting of the membranes with glyceraldehyde-3-phosphate-dehydrogenase (GAPDH) or $\beta$-tubulin. Representative Western blots are shown for 3-mercaptopyruvate sulfurtransferase (3-MST (a)), cystathionine- $\gamma$-lyase (CSE (b)), cystathionine- $\beta$-synthetase (CBS (c)), and $\beta$-tubulin (lower panel in a) and GAPDH (lower panel in $\mathbf{b}$ and $\mathbf{c}$ ). Densitometric analysis of all samples were normalized to GAPDH and expressed as fold induction for CBS (d). Graphs represent means \pm SEM, $n=6$ /group. ANOVA (StudentNewman-Keuls post hoc test), ${ }^{\S} P<0.05$ vs. LPS $+\mathrm{H}_{2} \mathrm{~S}$ treated group. 
or a combination of both (Fig. 3a, b). However, LPS instillation induced CBS expression which was absent in the presence of $\mathrm{H}_{2} \mathrm{~S}$ inhalation (Fig. 3c, d).

\section{Effect of Hydrogen Sulfide on Heat Shock Protein Expression}

Other important pathways in the prevention of LPSinduced ALI are ascribed to the induction of stress protein signaling, e.g., hemoxygenase-1 (HO-1) and heat shock protein 70 (HSP70) [3]. HO-1 protein expression was not affected by LPS and/or additional $\mathrm{H}_{2} \mathrm{~S}$ treatment (Fig. 4a). HSP70 protein expression was markedly reduced in lung tissue of LPS $+\mathrm{H}_{2} \mathrm{~S}$ mice, compared to all other groups (Fig. 4b, c).

\section{Effect of Hydrogen Sulfide on MAP Kinase Expression}

Because regulation of MAP kinase signaling has been shown to confer protection against LPS-induced ALI [14], we evaluated lung tissue protein expression of phosphorylated JNK, ERK1/2, and p38 by Western blotting. Phosphorylation of JNK was slightly reduced in the LPS $+\mathrm{H}_{2} \mathrm{~S}$ group compared to all other groups (Fig. 5a, b). In contrast to control and control $+\mathrm{H}_{2} \mathrm{~S}$ animals, pERK1/2 protein expression was elevated after LPS treatment in the LPS and LPS $+\mathrm{H}_{2} \mathrm{~S}$ group (Fig. 5c, d). However, statistical analysis of both pJNK and $\mathrm{pERK} 1 / 2$ did not reveal any differences. Expression of phosphorylated p38 MAPK (pp38 MAPK) was reduced by $\mathrm{H}_{2} \mathrm{~S}$ inhalation, both in the presence and absence of LPS, while LPS alone had no influence on pp38 MAPK expression as compared to non-treated control animals (Fig. 5e, f).

\section{Effect of Hydrogen Sulfide on Reactive Oxygen Species Production}

In LPS-induced ALI, the generation of ROS plays an essential role in the promotion of lung damage and inflammation [15]. We therefore evaluated, whether $\mathrm{H}_{2} \mathrm{~S}$ affects ROS production in our model of pulmonary inflammation. DHE staining of lung tissue slices revealed that in contrast to control or control $+\mathrm{H}_{2} \mathrm{~S}$ treated animals, LPS instillation alone caused significant ROS production (Fig. 6a "LPS"). This effect was prevented by additional $\mathrm{H}_{2} \mathrm{~S}$ inhalation (Fig. 6a "LPS $+\mathrm{H}_{2} \mathrm{~S}$ "). Quantification by densitometric analysis of all experimental groups and animals confirmed these results (Fig. 6b). a

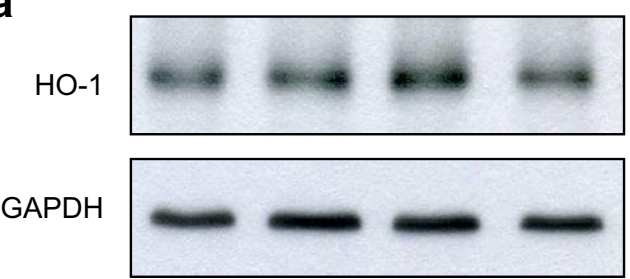

b

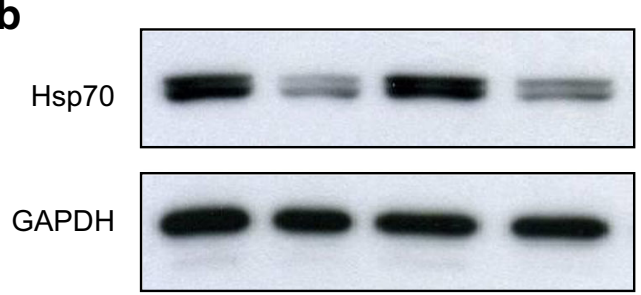

C

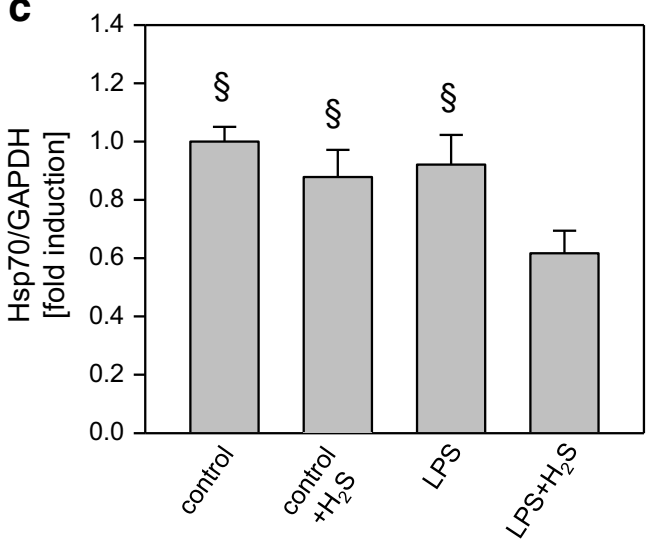

Fig. 4. Effect of hydrogen sulfide on heat shock protein expression. As controls, mice received phosphate-buffered saline (PBS, intranasally) and were kept in room air (control) or in $80 \mathrm{ppm} \mathrm{H}_{2} \mathrm{~S}$ (control $+\mathrm{H}_{2} \mathrm{~S}$ ) for $6 \mathrm{~h}$. LPS-treated mice (LPS i.n.) were either kept in room air (LPS) or in $80 \mathrm{ppm} \mathrm{H}_{2} \mathrm{~S}$ (LPS $+\mathrm{H}_{2} \mathrm{~S}$ ) for $6 \mathrm{~h}$. Lung samples were taken from the upper right lobe for Western blot analysis. Normalization in order to control equal protein loading was performed by stripping and re-blotting of the membranes with glyceraldehyde-3-phosphate-dehydrogenase (GAPDH). Representative Western blots are shown for hemoxygenase-1 (HO-1 (a)) and heat shock protein 70 (Hsp70 (b)) and GAPDH (lower panel in a and b). Densitometric analysis of all samples were normalized to GAPDH and expressed as fold induction for Hsp70 (c). Graphs represent means \pm SEM, $n=6$ /group. ANOVA (Student-Newman-Keuls post hoc test); ${ }^{\S} P<0.05 v s$. LPS $+\mathrm{H}_{2} \mathrm{~S}$ group.

\section{Effect of Hydrogen Sulfide on NADPH Oxidases}

Because the enzymatic generation of superoxide by NADPH oxidases (Nox) reflects the major source of ROS, we next investigated lung tissue protein expression of Nox1, Nox4, and Nox2, all previously 

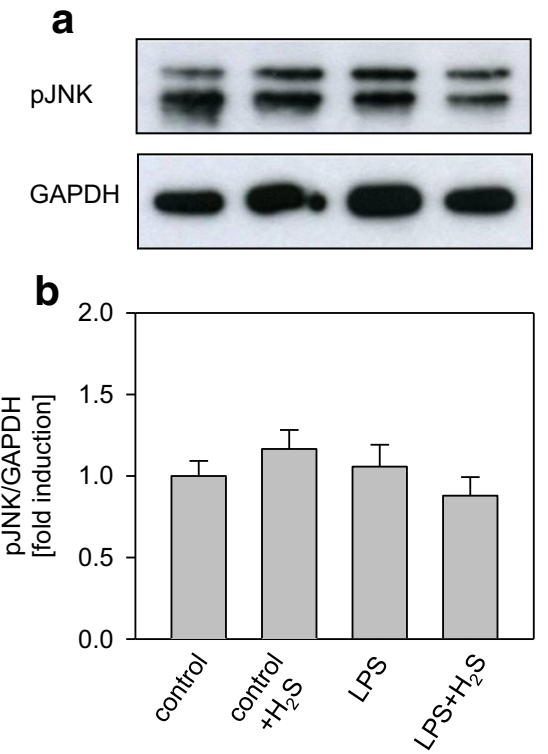
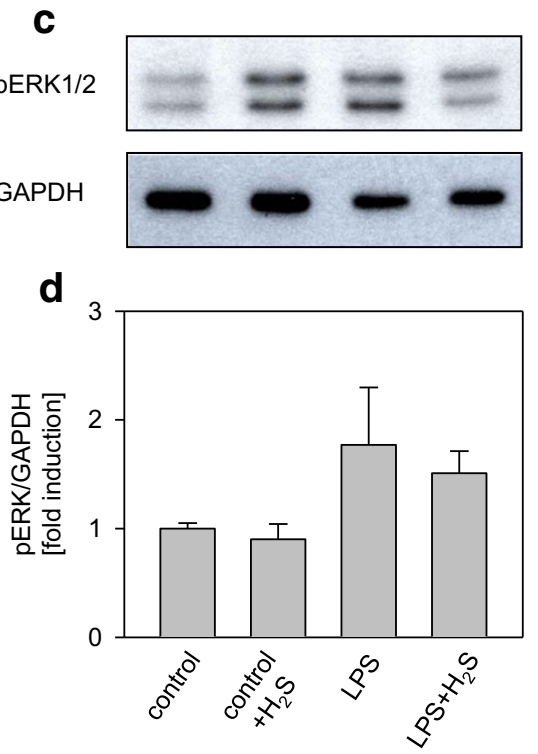

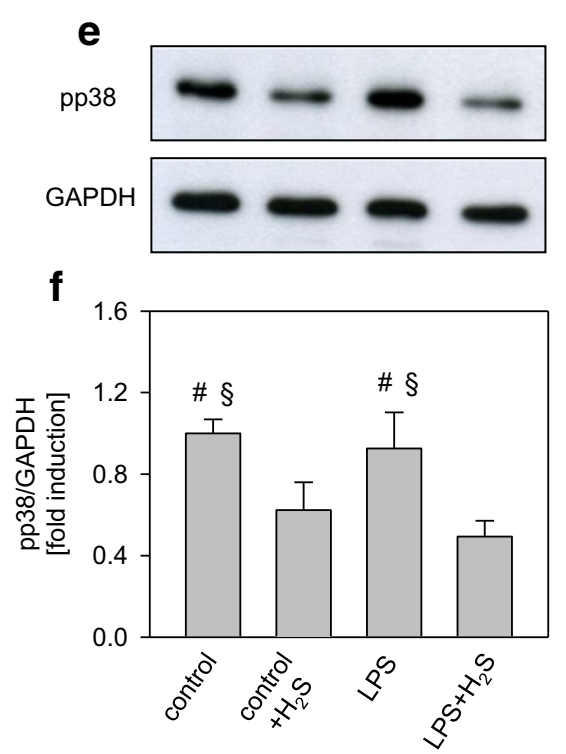

Fig. 5. Effect of hydrogen sulfide on MAP kinase expression. As controls, mice received phosphate-buffered saline (PBS, intranasally) and were kept in room air (control) or in $80 \mathrm{ppm} \mathrm{H}_{2} \mathrm{~S}$ (control $+\mathrm{H}_{2} \mathrm{~S}$ ) for $6 \mathrm{~h}$. LPS-treated mice (LPS i.n.) were either kept in room air (LPS) or in $80 \mathrm{ppm} \mathrm{H}_{2} \mathrm{~S}$ (LPS $+\mathrm{H}_{2} \mathrm{~S}$ ) for $6 \mathrm{~h}$. Lung samples were taken from the upper right lobe for Western blot analysis. Normalization in order to control equal protein loading was performed by stripping and re-blotting of the membranes with glyceraldehyde-3-phosphate-dehydrogenase (GAPDH). Representative Western blots are shown for pJNK (a), pERK1/2 (c), and pp38 (e) and GAPDH (lower panel in a, c, e). Densitometric analysis of all samples were normalized to GAPDH and expressed as fold induction for $\mathrm{pJNK}(\mathbf{b}), \mathrm{pERK} 1 / 2$ (d), and pp38 (f). Graphs represent means $\pm \mathrm{SEM}, n=6 /$ group. ANOVA (Student-Newman-Keuls post hoc test); ${ }^{\#} P<0.05$ vs. control $+\mathrm{H}_{2} \mathrm{~S}$ group; ${ }^{\S} P<0.05$ vs. LPS $+\mathrm{H}_{2} \mathrm{~S}$ group.

described to be expressed in the lungs and to be involved in ROS mediated organ damage in several injury models [15]. Neither LPS nor $\mathrm{H}_{2} \mathrm{~S}$ affected the expression of Nox1 or Nox4 (Fig. 7a, b). In contrast,
LPS induced Nox2 as compared to the control. $\mathrm{H}_{2} \mathrm{~S}$ inhalation, irrespective of LPS instillation, markedly reduced Nox2 protein expression in lung tissue (Fig. $7 \mathrm{c}, \mathrm{d})$. 


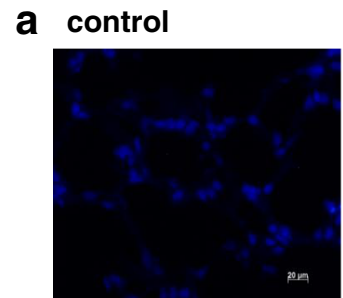

control+ $+\mathrm{H}_{2} \mathrm{~S}$
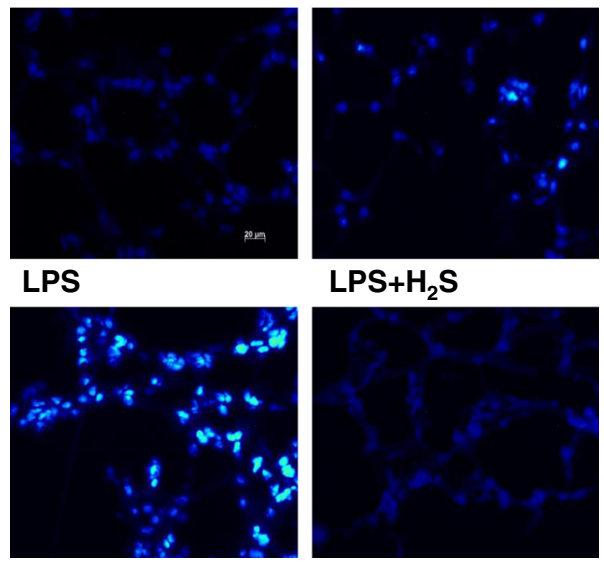

LPS $+\mathrm{H}_{2} \mathrm{~S}$
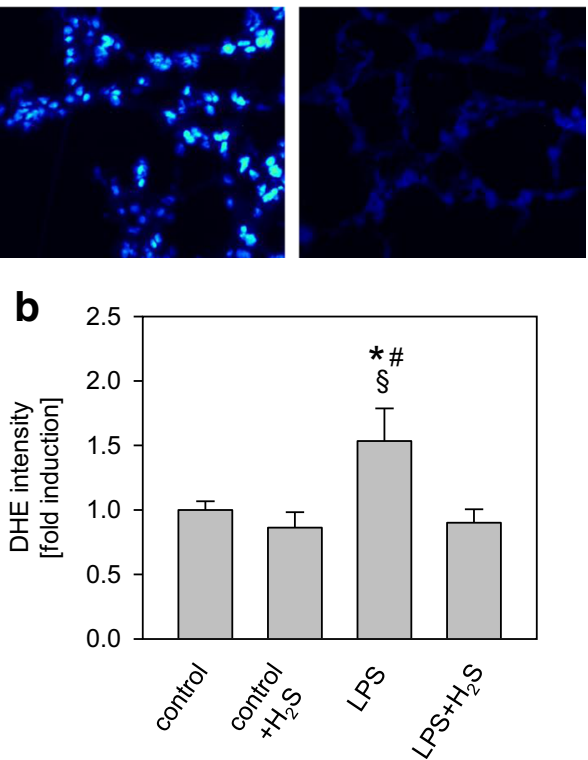

Fig. 6. Effect of hydrogen sulfide on reactive oxygen species production. As controls, mice received phosphate-buffered saline (PBS, intranasally) and were kept in room air (control) or in $80 \mathrm{ppm} \mathrm{H}_{2} \mathrm{~S}$ (control $+\mathrm{H}_{2} \mathrm{~S}$ ) for $6 \mathrm{~h}$. LPS-treated mice (LPS i.n.) were either kept in room air (LPS) or in $80 \mathrm{ppm}_{2} \mathrm{~S}$ (LPS $+\mathrm{H}_{2} \mathrm{~S}$ ) for $6 \mathrm{~h}$. Sections from the right lung lobe were stained by dihydroethidium (DHE). Representative pictures are shown for each experimental group as indicated (magnification $=200 \times($ a) $)$. DHE fluorescence intensity was measured and expressed as fold induction compared to PBS + air group (e). Graphs represent means \pm SEM, $n=6$ /group. ANOVA (Student-Newman-Keuls post hoc test); $* P<0.05$ vs. control group; ${ }^{\#} P<0.05$ vs. control + $\mathrm{H}_{2} \mathrm{~S}$ group; ${ }^{\S} P<0.05$ vs. LPS $+\mathrm{H}_{2} \mathrm{~S}$ group.

\section{DISCUSSION}

Acute lung injury resulting from lung infection remains a major problem in intensive care units and is associated with high morbidity and mortality rates. Despite modern antibiotics and supportive treatment, at present, specific therapeutic options in order to improve lung injury are lacking. In this respect, we have demonstrated recently that inhaled $\mathrm{H}_{2} \mathrm{~S}$ in low dose can efficiently protect from
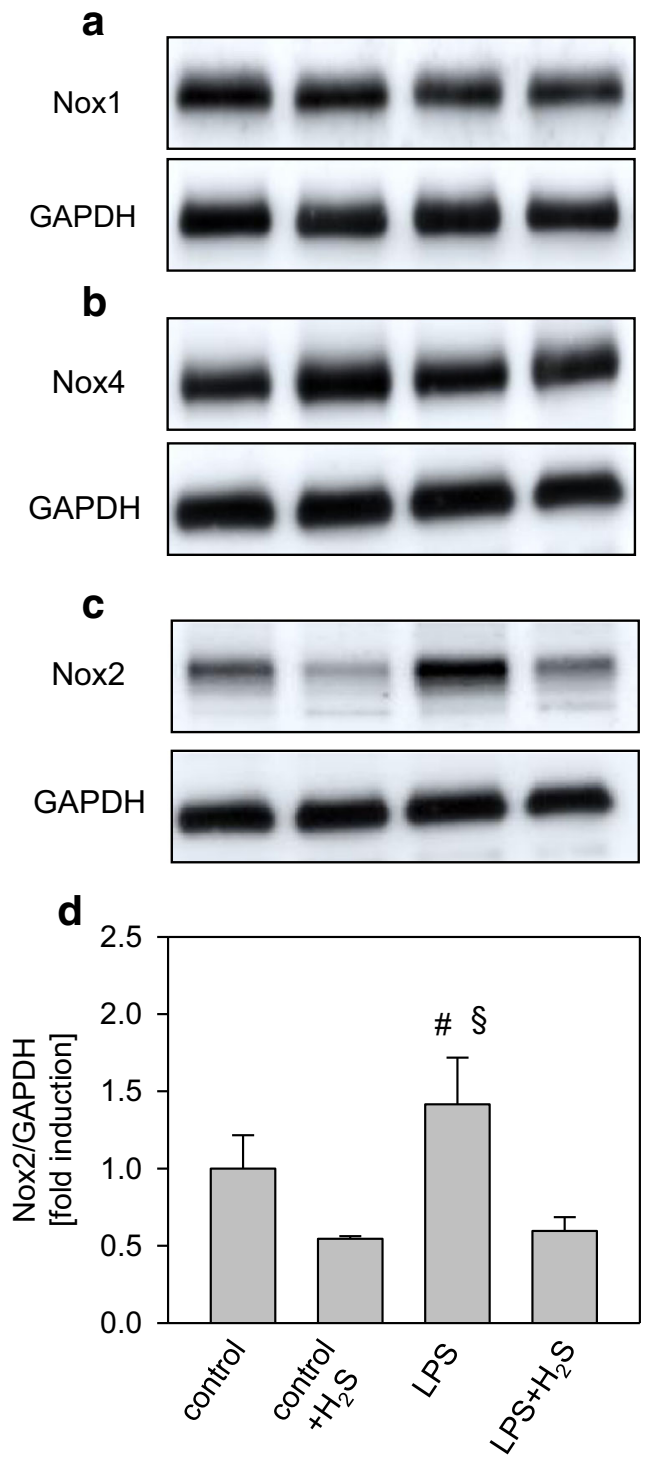

Fig. 7. Effect of hydrogen sulfide on NADPH oxidase expression. As controls, mice received phosphate-buffered saline (PBS, intranasally) and were kept in room air (control) or in $80 \mathrm{ppm} \mathrm{H}_{2} \mathrm{~S}$ (control $+\mathrm{H}_{2} \mathrm{~S}$ ) for $6 \mathrm{~h}$. LPS-treated mice (LPS i.n.) were either kept in room air (LPS) or in $80 \mathrm{ppm} \mathrm{H}_{2} \mathrm{~S}$ (LPS $+\mathrm{H}_{2} \mathrm{~S}$ ) for $6 \mathrm{~h}$. Lung samples were taken from the upper right lobe for Western blot analysis. Normalization in order to control equal protein loading was performed by stripping and re-blotting of the membranes with glyceraldehyde-3-phosphate-dehydrogenase (GAPDH). Representative Western blots are shown for Nox1 (a), Nox4 (b), Nox2 (c), and GAPDH (lower panel in a-c). Densitometric analysis of all samples were normalized to GAPDH and expressed as fold induction for Nox2 (d). Graphs represent means \pm SEM, $n=6$ /group. ANOVA on ranks (Dunn's post hoc test); ${ }^{\#} P<0.05$ vs. control $+\mathrm{H}_{2} \mathrm{~S}$ group; ${ }^{\S} P<0.05$ vs. $\mathrm{LPS}+\mathrm{H}_{2} \mathrm{~S}$ group. 
ALI in a mouse model of pulmonary inflammation [11]. However, the underlying molecular pathways affected by $\mathrm{H}_{2} \mathrm{~S}$ in this model remain elusive. In the present study, we show for the first time that $\mathrm{H}_{2} \mathrm{~S}$ mediates this protection via the inhibition of LPS-induced inflammatory and oxidative processes, which in turn may result in the observed organ protection.

\section{Hydrogen Sulfide Prevents Lung Damage by Inhibiting Inflammatory Processes}

We have previously shown that inhalation of $\mathrm{H}_{2} \mathrm{~S}$ averts the development of acute lung injury in a mouse model of LPS-induced acute lung injury [11]. In the current study, we again showed that LPS treatment clearly elevated histological signs of lung damage, e.g., alveolar wall thickening and ALI score as compared to controls. The effect was completely prevented by inhalation of $\mathrm{H}_{2} \mathrm{~S}$. Similar results have been obtained by us and others in mouse models of ventilator-induced lung injury $[8,16]$, hyperoxia-induced lung injury [9], or endotoxin-induced systemic inflammation [17], strongly underlining the lung protective capacity of $\mathrm{H}_{2} \mathrm{~S}$ treatment.

Lung injury as a response to LPS instillation has been clearly linked to the elicited inflammatory response [18]. Neutrophil migration into lung tissue and the release of pro-inflammatory cytokines like IL- $1 \beta$ have been shown to mediate ALI [19]. In our model, LPS treatment led to a vast transmigration of neutrophils into the bronchoalveolar fluid that was accompanied by an increased IL- $1 \beta$ secretion. $\mathrm{H}_{2} \mathrm{~S}$ inhalation on the other hand reduced both neutrophil recruitment and IL- $1 \beta$ release back to control levels. These findings are in line with recent publications demonstrating the anti-inflammatory properties of $\mathrm{H}_{2} \mathrm{~S}$ in in vivo lung injury models like pulmonary inflammation $[11,17]$, mechanical ventilation [8], hyperoxia [9], or acute pancreatitis [20], reflecting that $\mathrm{H}_{2} \mathrm{~S}$-induced lung protection is mediated by anti-inflammatory effects.

\section{Hydrogen Sulfide Inhibits CBS Expression in LPS- Induced ALI}

In the present study, we determined the impact of LPS instillation with or without $\mathrm{H}_{2} \mathrm{~S}$ inhalation on CSE, CBS, and 3-MST expression in the lung. These enzymes endogenously catalyze the synthesis of $\mathrm{H}_{2} \mathrm{~S}$ [21] and are thought to be involved in organ protective effects in organ injury models [22-25]. Especially the regulation of CSE has been shown to exert organ protective effects in models of acute lung injury induced by acute pancreatitis [22, 24], but also all three were proven to be involved in attenuation of acute kidney injury in rats [23]. Our data reveal that lung protein expression of 3-MST and CSE was neither affected by LPS nor by $\mathrm{H}_{2} \mathrm{~S}$ treatment. Although reduced CSE activity was attributed to LPS instillation in the rat lung [26, 27], we could not detect any effect on pulmonary CSE upon LPS challenge. In contrast, LPS instillation led to CBS induction that was prevented in the presence of $\mathrm{H}_{2} \mathrm{~S}$. Similar findings were reported in a recent publication of Wagner et al. [25]. Here, in a mouse model of blunt chest trauma, intravenous sulfide injection resulted in the attenuation of lung tissue apoptosis, which was accompanied by a reduced CBS and CSE protein expression [25]. Likewise, the application of exogenous $\mathrm{H}_{2} \mathrm{~S}$ in our model may initiate the suppression of CBS activity, either as a result of reduced inflammation or as a direct negative feedback mechanism. Nonetheless, we show for the first time, that local administration of $\mathrm{H}_{2} \mathrm{~S}$ regulates $\mathrm{CBS}$ expression in the lung, probably resulting in a reduction of endogenous $\mathrm{H}_{2} \mathrm{~S}$ production.

\section{Hydrogen Sulfide Prevents Heat Shock Protein Expression}

Heat shock proteins like HO-1 or HSP70 are induced due to various insults in vivo and in vitro. Upregulation of both HO-1 and HSP70 in lung tissue have been described as a result of LPS stimulation and are clearly involved in protection against inflammation [3]. However, in our model, protein expression of HO-1 was unaffected by both LPS stimulation and/or additional $\mathrm{H}_{2} \mathrm{~S}$ inhalation, while HSP70 expression was reduced due to the combination of LPS and $\mathrm{H}_{2} \mathrm{~S}$ treatment. Although we did not find a role for HO-1 in our model, the results of HSP70 are in line with our recent observations in a model of ventilator-induced lung injury, where $\mathrm{H}_{2} \mathrm{~S}$ treatment also clearly reduced HSP70 expression [8]. These results suggest that $\mathrm{H}_{2} \mathrm{~S}$ may limit proinflammatory stress signaling in LPS-induced ALI.

\section{Hydrogen Sulfide Prevents p38 MAP Kinase Signaling}

Another possible pathway involved in the observed lung protective effects of $\mathrm{H}_{2} \mathrm{~S}$ may be MAPK signaling. The regulation of MAPK activity has been reported to mediate inflammatory and/or oxidative effects upon infectious insults, thus augmenting lung injury [4, 28, 29]. Moreover, application of $\mathrm{H}_{2} \mathrm{~S}$ has been described to block MAPK signaling $[5,6,30]$. Inhibition of p38 activation by $\mathrm{H}_{2} \mathrm{~S}$ protected microglia from LPS-induced inflammation [5]. Both $\mathrm{p} 38$ and ERK $1 / 2$ expression were reduced upon $\mathrm{H}_{2} \mathrm{~S}$ application in PC-12 cells in a model of hypoxiainduced injury [6], and $\mathrm{H}_{2} \mathrm{~S}$ prevented endothelial cell 
damage in rats in an ischemia-reperfusion rat model by blocking p38 and JNK signaling [30]. Our results showed no major effect of LPS or $\mathrm{H}_{2} \mathrm{~S}$ on the regulation of $\mathrm{pJNK}$ or pERK1/2 expression. In contrast, phosphorylation of the p38 MAPK was significantly reduced by $\mathrm{H}_{2} \mathrm{~S}$ inhalation, although LPS alone showed no effect. A more recent study reported that $\mathrm{H}_{2} \mathrm{~S}$ treatment can inhibit p38 MAPK activation, leading to cellular protection in in vitro models of LPS-induced inflammation [5] or cobalt chloride-induced hypoxia [6]. Moreover, Sivarajah and co-workers demonstrated that the observed cardioprotective effects of $\mathrm{H}_{2} \mathrm{~S}$ in a rat model of regional myocardial ischemia-reperfusion injury were at least in part due to a reduction in p38 phosphorylation [31], suggesting that $\mathrm{H}_{2} \mathrm{~S}$-related decreases in pp38 may play a role in protection from LPSinduced lung injury in our model.

\section{Hydrogen Sulfide Prevents ROS Production and NADPH Oxidase 2 Signaling}

It is widely accepted that pro-inflammatory signaling involves the accumulation of reactive oxygen species. Likewise, acute lung injury in response to LPS treatment is characterized by excessive ROS production [32] and its suppression has been shown to protect lungs from LPSinduced ALI in several models [15]. $\mathrm{H}_{2} \mathrm{~S}$ can limit ROS either by directly scavenging free radicals, thus regulating oxidative signaling pathways, or by suppressing the inflammatory response [33]. Here, we clearly demonstrate that the vast ROS formation due to LPS challenge was prevented by $\mathrm{H}_{2} \mathrm{~S}$ treatment. These findings are in line with recent studies carried out both by ourselves and others in vivo as well as in vitro [9, 34-36]. For instance, in mouse model of hyperoxia-induced, ROS were reduced by $\mathrm{H}_{2} \mathrm{~S}$ application, which was accompanied by the inhibition of lung damage [9]. Likewise, in stimulated epithelial A549 cells [9], endothelial HUVEC cells [9], and RAW 264.7 macrophages [9], PC12 [35], and H9c2 [34] cells, application of $\mathrm{H}_{2} \mathrm{~S}$ suppressed ROS formation and prevented the development of an inflammatory response.

With respect to an underlying mechanism, LPSinduced production of ROS is mainly attributed to the enzymatic activity of NADPH oxidases [37]. Several Nox family members have been described [32]. Among these, the regulation of Nox1, Nox2, and Nox4 were associated with acute lung injury $[15,38,39]$. In addition, $\mathrm{H}_{2} \mathrm{~S}$ can reduce Nox expression in vivo and in vitro [40-42]. Our results show that neither LPS nor $\mathrm{H}_{2} \mathrm{~S}$ influenced Nox 1 or Nox4 regulation. In contrast, LPS-induced Nox2 expression was prevented in response to $\mathrm{H}_{2} \mathrm{~S}$ inhalation. The importance of Nox2 regulation in lung oxidative stress and inflammation has been shown more recently [38, 39]. For instance, Menden and co-workers found in human pulmonary microvascular endothelial cells that LPS-induced oxidative stress and proinflammatory signaling was regulated by Nox2 [39]. In another LPS mouse model, Gandhirajan et al. showed that blockade of Nox 2 signaling prevented vascular leakage and pulmonary edema in endothelial cells [38], suggesting that inhibition of Nox 2 expression in our model, probably followed by downregulation of MAPK signaling, may also inhibit the subsequent development of ALI.

\section{CONCLUSION}

In the present study, inhalation of hydrogen sulfide prevents LPS-induced acute lung injury. CBS, HSP70, p38, Nox2 protein expression, and ROS production were all modulated due to $\mathrm{H}_{2} \mathrm{~S}$ treatment. Although the results of the current study do not reveal the rate of contribution or a potential interaction between the signaling pathways, it appears reasonable that the anti-inflammatory effects of $\mathrm{H}_{2} \mathrm{~S}$-mediated lung protection are caused by limiting ROS production due to inhibition of Nox 2 and p38 MAPK signaling pathways.

\section{ACKNOWLEDGMENTS}

This study was supported by a grant from the Deutsche Forschungsgemeinschaft (Bonn, Germany) to Alexander Hoetzel (DFG HO 2464/3-1).

The authors thank E. Bodurova (Life Imaging Centre, Albert-Ludwigs-University Freiburg, Germany) for the excellent technical assistance.

Parts of the study have been published online as the doctoral thesis by Kornelia K. Zimmermann, 2013: "Die protektive Wirkung Schwefelwasserstoffs bei oxidativer und inflammatorischer Lungenschädigung" [German] https://www.freidok.uni-freiburg.de/dnb/download/9093.

Authors' Contributions KKZ helped to conduct the study and analyzed the data; KMS helped to conduct the study; PMI helped to design and conduct the study and to analyze the data; HE helped to analyze the data and critically revised the manuscript; SGS helped to conduct the study and analyzed the data; $\mathrm{AH}$ helped to design and conduct the study, to analyze the data and to write the manuscript; and SF designed and conducted the study, analyzed the data, and wrote the manuscript. All authors read and approved the final manuscript. 


\section{COMPLIANCE WITH ETHICAL STANDARDS}

Competing Interests. This study was supported by a grant from the Deutsche Forschungsgemeinschaft (Bonn, Germany) to Alexander Hoetzel (DFG HO 2464/3-1). All other authors declare that they have no competing interests.

Ethical Approval. All applicable international, national, and/or institutional guidelines for the care and use of animals were followed. All procedures performed in studies involving animals were in accordance with the ethical standards of the institution or practice at which the studies were conducted (Ethics Committee University of Freiburg and Regierungspräsidium Freiburg, Freiburg, Germany, Permission No. G-07/25).

\section{REFERENCES}

1. Martin, G.S., D.M. Mannino, S. Eaton, and M. Moss. 2003. The epidemiology of sepsis in the United States from 1979 through 2000. The New England Journal of Medicine 348: $1546-1554$.

2. Matute-Bello, G., C.W. Frevert, and T.R. Martin. 2008. Animal models of acute lung injury. American Journal of Physiology. Lung Cellular and Molecular Physiology 295: L379-L399.

3. Wheeler, D.S., and H.R. Wong. 2007. Heat shock response and acute lung injury. Free Radical Biology \& Medicine 42: 1-14.

4. Arndt, P.G., S.K. Young, and G.S. Worthen. 2005. Regulation of lipopolysaccharide-induced lung inflammation by plasminogen activator Inhibitor-1 through a JNK-mediated pathway. Journal of Immunology 175: 4049-4059.

5. Hu, L.F., P.T. Wong, P.K. Moore, and J.S. Bian. 2007. Hydrogen sulfide attenuates lipopolysaccharide-induced inflammation by inhibition of p38 mitogen-activated protein kinase in microglia. Journal of Neurochemistry 100: 1121-1128.

6. Lan, A., X. Liao, L. Mo, C. Yang, Z. Yang, X. Wang, F. Hu, P. Chen, J. Feng, D. Zheng, and L. Xiao. 2011. Hydrogen sulfide protects against chemical hypoxia-induced injury by inhibiting ROSactivated ERK1/2 and p38MAPK signaling pathways in PC1 2 cells. PLoS One 6: e25921.

7. Biermann, J., W.A. Lagreze, N. Schallner, C.I. Schwer, and U. Goebel. 2011. Inhalative preconditioning with hydrogen sulfide attenuated apoptosis after retinal ischemia/reperfusion injury. Molecular Vision 17: 1275-1286.

8. Faller, S., S.W. Ryter, A.M. Choi, T. Loop, R. Schmidt, and A. Hoetzel. 2010. Inhaled hydrogen sulfide protects against ventilatorinduced lung injury. Anesthesiology 113: 104-115.

9. Faller, S., S.G. Spassov, K.K. Zimmermann, S.W. Ryter, H. Buerkle, T. Loop, R. Schmidt, K.M. Strosing, and A. Hoetzel. 2013. Hydrogen sulfide prevents hyperoxia-induced lung injury by downregulating reactive oxygen species formation and angiopoietin-2 release. Current Pharmaceutical Design 19: 2715-2721.
10. Li, T., B. Zhao, C. Wang, H. Wang, Z. Liu, W. Li, H. Jin, C. Tang, and J. Du. 2008. Regulatory effects of hydrogen sulfide on IL-6, IL8 and IL-10 levels in the plasma and pulmonary tissue of rats with acute lung injury. Exp Biol Med (Maywood). 233: 1081-1087.

11. Faller, S., K.K. Zimmermann, K.M. Strosing, H. Engelstaedter, H. Buerkle, R. Schmidt, S.G. Spassov, and A. Hoetzel. 2012. Inhaled hydrogen sulfide protects against lipopolysaccharide-induced acute lung injury in mice. Medical Gas Research 2: 26.

12. Faller, S., K.M. Strosing, S.W. Ryter, H. Buerkle, T. Loop, R. Schmidt, and A. Hoetzel. 2012. The volatile anesthetic isoflurane prevents ventilator-induced lung injury via phosphoinositide 3-kinase/Akt signaling in mice. Anesthesia and Analgesia 114: 747756.

13. Madden, J.A., S.B. Ahlf, M.W. Dantuma, K.R. Olson, and D.L. Roerig. 2012. Precursors and inhibitors of hydrogen sulfide synthesis affect acute hypoxic pulmonary vasoconstriction in the intact lung. Journal of Applied Physiology 112 (3): 411-418.

14. Zhang, T.Z., S.H. Yang, J.F. Yao, J. Du, and T.H. Yan. 2015. Sangxingtang inhibits the inflammation of LPS-induced acute lung injury in mice by down-regulating the MAPK/NF-kappaB pathway. Chinese Journal of Natural Medicines 13: 889-895.

15. Carnesecchi, S., J.C. Pache, and C. Barazzone-Argiroffo. 2012. NOX enzymes: potential target for the treatment of acute lung injury. Cellular and Molecular Life Sciences 69: 2373-2385.

16. Francis, R.C., K. Vaporidi, K.D. Bloch, F. Ichinose, and W.M. Zapol. 2011. Protective and detrimental effects of sodium sulfide and hydrogen sulfide in murine ventilator-induced lung injury. Anesthesiology 115: 1012-1021.

17. Tokuda, K., K. Kida, E. Marutani, E. Crimi, M. Bougaki, A. Khatri, H. Kimura, and F. Ichinose. 2012. Inhaled hydrogen sulfide prevents endotoxin-induced systemic inflammation and improves survival by altering sulfide metabolism in mice. Antioxidants \& Redox Signaling 17: 11-21.

18. Hasan, B., F.S. Li, A. Siyit, E. Tuyghun, J.H. Luo, H. Upur, and A. Ablimit. 2014. Expression of aquaporins in the lungs of mice with acute injury caused by LPS treatment. Respiratory Physiology \& Neurobiology 200C: 40-45.

19. Li, L., G. Rossoni, A. Sparatore, L.C. Lee, P. Del Soldato, and P.K. Moore. 2007. Anti-inflammatory and gastrointestinal effects of a novel diclofenac derivative. Free Radical Biology \& Medicine 42: 706-719.

20. Sidhapuriwala, J.N., S.W. Ng, and M. Bhatia. 2009. Effects of hydrogen sulfide on inflammation in caerulein-induced acute pancreatitis. Journal Inflammation (London) 6: 35.

21. Huang, C.W., and P.K. Moore. 2015. H2S synthesizing enzymes: biochemistry and molecular aspects. Handbook of Experimental Pharmacology 230: 3-25.

22. Ang, A.D., J. Rivers-Auty, A. Hegde, I. Ishii, and M. Bhatia. 2013. The effect of CSE gene deletion in caerulein-induced acute pancreatitis in the mouse. American Journal of Physiology. Gastrointestinal and Liver Physiology 305: G712-G721.

23. Dugbartey, G.J., F. Talaei, M.C. Houwertjes, M. Goris, A.H. Epema, H.R. Bouma, and R.H. Henning. 2015. Dopamine treatment attenuates acute kidney injury in a rat model of deep hypothermia and rewarming - the role of renal H2S-producing enzymes. European Journal of Pharmacology 769: 225-233.

24. Qu, Z., Y. Jiang, B.Q. Wu, Y.F. Duan, Z.D. Sun, and G.H. Luo. 2014. Cystathionine-gamma-lyase inhibitor attenuates acute lung injury induced by acute pancreatitis in rats. Archives of Medical Science 10: 825-829.

25. Wagner, F., A. Scheuerle, S. Weber, B. Stahl, O. McCook, M.W. Knoferl, M. Huber-Lang, D.H. Seitz, J. Thomas, P. Asfar, C. Szabo, P. Moller, F. Gebhard, M. Georgieff, E. Calzia, P. Radermacher, and 
K. Wagner. 2011. Cardiopulmonary, histologic, and inflammatory effects of intravenous $\mathrm{Na} 2 \mathrm{~S}$ after blunt chest trauma-induced lung contusion in mice. The Journal of Trauma 71: 1659-1667.

26. Zhou, X.H., P. Wei, X.L. Huang, and Y.L. Ling. 2009. Role of endogenous and exogenous hydrogen sulfide in acute lung injury induced by LPS in rats. Zhongguo Ying Yong Sheng Li Xue Za Zhi 25: 289-294.

27. Zhou, X.H., X.L. Huang, P. Wei, F.J. Tian, and Y.L. Ling. 2009. Role of hydrogen sulfide/cystathionine-gamma-lyase system in acute lung injury induced by lipopolysaccharide in rats. Zhongguo Wei Zhong Bing Ji Jiu Yi Xue 21: 199-202.

28. Asaduzzaman, M., Y. Wang, and H. Thorlacius. 2008. Critical role of p38 mitogen-activated protein kinase signaling in septic lung injury. Critical Care Medicine 36: 482-488.

29. Schuh, K., and A. Pahl. 2009. Inhibition of the MAP kinase ERK protects from lipopolysaccharide-induced lung injury. Biochemical Pharmacology 77: 1827-1834.

30. Issa, K., A. Kimmoun, S. Collin, F. Ganster, S. Fremont-Orlowski, P. Asfar, P.M. Mertes, and B. Levy. 2013. Compared effects of inhibition and exogenous administration of hydrogen sulphide in ischaemia-reperfusion injury. Critical Care 17: R129.

31. Sivarajah, A., M. Collino, M. Yasin, E. Benetti, M. Gallicchio, E. Mazzon, S. Cuzzocrea, R. Fantozzi, and C. Thiemermann. 2009. Anti-apoptotic and anti-inflammatory effects of hydrogen sulfide in a rat model of regional myocardial I/R. Shock 31: 267-274.

32. Lee, I.T., and C.M. Yang. 2012. Role of NADPH oxidase/ROS in pro-inflammatory mediators-induced airway and pulmonary diseases. Biochemical Pharmacology 84: 581-590.

33. Stein, A., and S.M. Bailey. 2013. Redox biology of hydrogen sulfide: implications for physiology, pathophysiology, and pharmacology. Redox Biology 1: 32-39.

34. Dong, X.B., C.T. Yang, D.D. Zheng, L.Q. Mo, X.Y. Wang, A.P. Lan, F. Hu, P.X. Chen, J.Q. Feng, M.F. Zhang, and X.X. Liao. 2012. Inhibition of ROS-activated ERK1/2 pathway contributes to the protection of $\mathrm{H} 2 \mathrm{~S}$ against chemical hypoxia-induced injury in H9c2 cells. Molecular and Cellular Biochemistry 362: 149-157.
35. Lan, A., W. Xu, H. Zhang, X. Hua, D. Zheng, R. Guo, N. Shen, F. $\mathrm{Hu}$, J. Feng, and D. Liu. 2013. Inhibition of ROS-activated p38MAPK pathway is involved in the protective effect of $\mathrm{H} 2 \mathrm{~S}$ against chemical hypoxia-induced inflammation in PC12 cells. Neurochemical Research 38: 1454-1466.

36. Yu, Q., Z. Lu, L. Tao, L. Yang, Y. Guo, Y. Yang, X. Sun, and Q. Ding. 2015. ROS-dependent neuroprotective effects of NaHS in ischemia brain injury involves the PARP/AIF pathway. Cellular Physiology and Biochemistry 36: 1539-1551.

37. Kratzer, E., Y. Tian, N. Sarich, T. Wu, A. Meliton, A. Leff, and A.A. Birukova. 2012. Oxidative stress contributes to lung injury and barrier dysfunction via microtubule destabilization. American Journal of Respiratory Cell and Molecular Biology 47: 688-697.

38. Gandhirajan, R.K., S. Meng, H.C. Chandramoorthy, K. Mallilankaraman, S. Mancarella, H. Gao, R. Razmpour, X.F. Yang, S.R. Houser, J. Chen, W.J. Koch, H. Wang, J. Soboloff, D.L. Gill, and M. Madesh. 2013. Blockade of NOX2 and STIM1 signaling limits lipopolysaccharide-induced vascular inflammation. The Journal of Clinical Investigation 123: 887-902.

39. Menden, H., E. Tate, N. Hogg, and V. Sampath. 2013. LPS-mediated endothelial activation in pulmonary endothelial cells: role of Nox2dependent IKK-beta phosphorylation. American Journal of Physiology. Lung Cellular and Molecular Physiology 304: L445-L455.

40. Li, H.D., Z.R. Zhang, Q.X. Zhang, Z.C. Qin, D.M. He, and J.S. Chen. 2013. Treatment with exogenous hydrogen sulfide attenuates hyperoxia-induced acute lung injury in mice. European Journal of Applied Physiology 113: 1555-1563.

41. Tyagi, N., K.S. Moshal, U. Sen, T.P. Vacek, M. Kumar, W.M. Hughes Jr., S. Kundu, and S.C. Tyagi. 2009. H2S protects against methionine-induced oxidative stress in brain endothelial cells. Antioxidants \& Redox Signaling 11: 25-33.

42. Zheng, D., S. Dong, T. Li, F. Yang, X. Yu, J. Wu, X. Zhong, Y. Zhao, L. Wang, C. Xu, F. Lu, and W. Zhang. 2015. Exogenous hydrogen sulfide attenuates cardiac fibrosis through reactive oxygen species signal pathways in experimental diabetes mellitus models. Cellular Physiology and Biochemistry 36: 917-929. 